\title{
Creating a virtual device for processing the results of sorption measurements in the study of zinc oxide nanorods
}

\author{
E. V. Maraeva ${ }^{a^{*}}$, N. V. Permiakov ${ }^{a}$, Y. Y. Kedruk ${ }^{b}$, \\ L. V. Gritsenkob, Kh. A. Abdullin \\ ${ }^{a}$ Department of Micro-and Nanoelectronics, Saint Petersburg Electrotechnical \\ University «LETI», Prof. Popova St., 5, Saint Petersburg 197376, Russsia \\ ${ }^{b}$ Satbayev University, Satpaeva St, 22, Almaty 050013, Kazakhstan \\ c al-Farabi Kazakh National University, al-Farabi Ave, 71, Almaty 050040, \\ Kazakhstan \\ *email: jenvmar@mail.ru
}

\begin{abstract}
The work is devoted to the creation of a virtual device (computer program) for processing the results of sorption analysis of nanomaterials, including for estimating the size of nanoparticles based on the specific surface area. The obtained evaluation results were compared with the scanning electron microscopy data. Photocatalytically active zinc oxide samples were chosen as the object of the study.
\end{abstract}

Keywords: zinc oxide; nanorods; sorption measurements; virtual device;

Received: 15.10.2020. Accepted: 09.12.2020. Published:30.12.2020.

(C) Maraeva E. V., Permiakov N. V., Kedruk Y. Y., Gritsenko L. V., Abdullin Kh. A., 2020

\section{Introduction}

Sorption analysis methods, as a rule, are used to characterize porous materials at the mesoporous (diameter $2-50 \mathrm{~nm}$ ), as well as microporous (diameter $\leq 2 \mathrm{~nm}$ ) levels. However, such a characteristic of the material as the specific surface area, determined on the basis of analysis of inert gas adsorption isotherms, in some cases characterizes not only the surface development due to the porous structure of the material, but also the surface of the particles of the fragmented phase of the dispersed system.

In this case, the data of a sorption study provide an express estimation of the sizes of nanoparticles, while the sorption analysis methods have some advantages over other methods. Such advantages are the possibility of studying a large amount of material in one step (unlike local methods where individual sections of the sample are analyzed and the results of the study are averaged), as well as the possibility of studying materials with high roughness, where other methods of surface analysis are not applicable or undesirable.

The aim of this work was to create a virtual device (computer program) for processing the results of sorption analysis of nanomaterials, including for assessing the size of nanoparticles based on the specific surface area determined by the standard BET method. As the object under study 
photocatalytically active zinc oxide samples [1] obtained by annealing zinc acetate di-

\section{Experimental}

$\mathrm{ZnO}$ nanoparticles (NPs) were synthesized by annealing zinc acetate dihydrate $\left(\mathrm{CH}_{3} \mathrm{COO}\right)_{2} \mathrm{Zn} \cdot 2 \mathrm{H}_{2} \mathrm{O}$ in a muffle furnace at temperatures of $400{ }^{\circ} \mathrm{C}$ and $700{ }^{\circ} \mathrm{C}$; the annealing time was 2,4 , and 10 hours. Zinc acetate was placed in a ceramic crucible covered with a ceramic lid. The mass of the obtained ZnO NPs sample was from a quarter to a third of the mass of zinc acetate. According to [2], the main weight loss occurs due to the combustion of acetone $\left(\left(\mathrm{CH}_{3}\right)_{2} \mathrm{CO}\right)$ and carbon dioxide $\left(\mathrm{CO}_{2}\right)$ in the precursor.

Particle sizes were determined based on the processing of sorption analysis

\section{Results and discussion}

In this work, five types of zinc oxide samples were investigated. The parameters of their heat treatment and the results of determining the specific surface area are shown in Table 1.

The data presented in Fig. 1 were the initial ones for determining the particle sizes, which was carried out in two stages. The first stage was to determine the specific surface according to the method

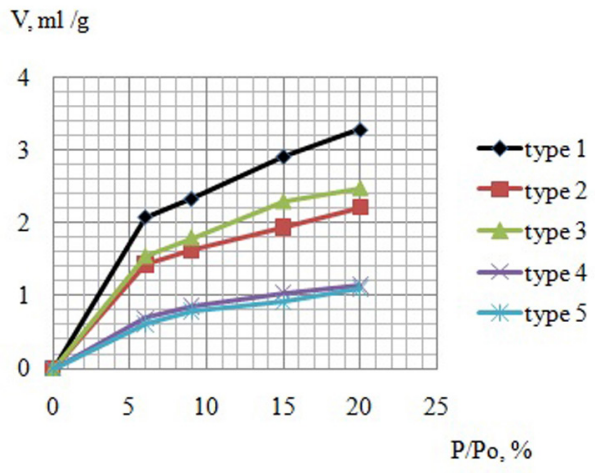

Fig. 1. Plots of adsorption isotherms for samples $1-5$ hydrate $\left(\mathrm{CH}_{3} \mathrm{COO}\right)_{2} \mathrm{Zn} \cdot 2 \mathrm{H}_{2} \mathrm{O}$ in a muffle furnace were chosen.

data implemented on a Sorbi MS device, which allows one to study the parameters of the porous structure of nanomaterials [3], including determining the size distribution of mesopores and specific surface area. The obtained evaluation results were compared with the data of scanning electron microscopy.

To develop a virtual program, the LabView environment was used. The input to the analysis was a fragment of the adsorption isotherm of the selected inert gas on the test sample, the density of the test material, and the aspect ratio of the nanorods.

of Brunauer, Emmett, Teller ( $S_{\mathrm{BET}}$ Table 1$)$. At the second stage, the average particle size was determined from the specific surface area in the framework of the model that the particles are nanorods with a given ratio of length and diameter $L / D$. The $L / D$ ratio was selected on the basis of processing the experimental data of scanning electron microscopy, individually for each series of samples obtained by varying the annealing modes.

Table 1

$\mathrm{ZnO}$ specific surface area vs. heat treatment temperature and duration

\begin{tabular}{c|c|c|c}
\hline \multirow{2}{*}{$№$} & \multicolumn{2}{|c|}{$\begin{array}{c}\text { Heat treatment } \\
\text { parameters }\end{array}$} & \multirow{2}{*}{$S_{\text {BET }} \mathrm{m}^{2} / \mathrm{g}$} \\
\cline { 2 - 3 } & $T,{ }^{\circ} \mathrm{C}$ & $t$, hours & \\
\hline 1 & 400 & 2 & 13 \\
\hline 2 & 400 & 4 & 9 \\
\hline 3 & 400 & 10 & 10 \\
\hline 4 & 700 & 2 & 5 \\
\hline 5 & 700 & 4 & 5 \\
\hline
\end{tabular}


Fig. 1 shows the nitrogen adsorption isotherm for a series of zinc oxide samples $1-5$.

According to the data of scanning electron microscopy, the particles had a rodlike shape, and it was noted that the samples synthesized at $400{ }^{\circ} \mathrm{C}$ had a shorter length and thickness than those obtained at $700{ }^{\circ} \mathrm{C}$ with the corresponding annealing duration. For example, Fig. 2 shows SEM images of the surface of a samples 1 and 4, and Table 2 shows the results of processing the experimental SEM data for all types of samples.

The diameter of the nanorods was calculated according to the formula:

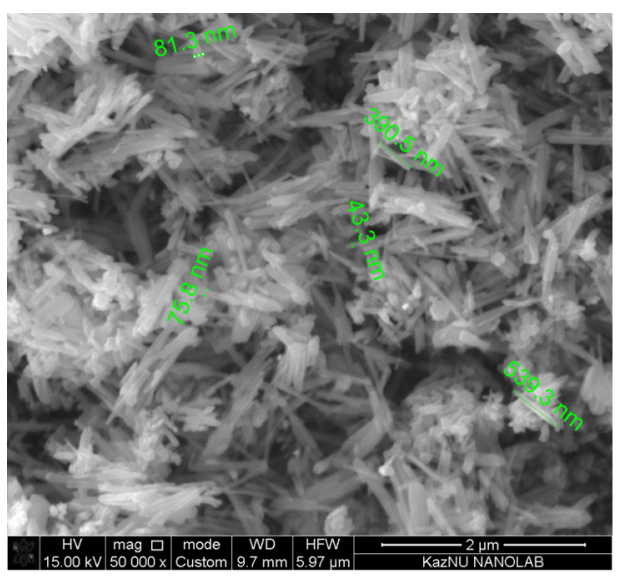

a

$$
D=\frac{4 n+2}{\rho \cdot n \cdot S_{\mathrm{BET}}}
$$

where $n$ - the aspect ratio, $\rho$ - the density, $S_{\mathrm{BET}}-$ specific surface area of the material.

Fig. 3 shows for example a fragment of a block diagram (graphic code) of a program that calculates a fragment of nanorods for a given aspect ratio $n$.

To calculate the size of the core particles by the sorption method, we used the approximation that the diameter of the $\operatorname{rod} D$ refers to its height $L$ according to the data presented in Table 2. The results of the sorption analysis are presented in Table 3.

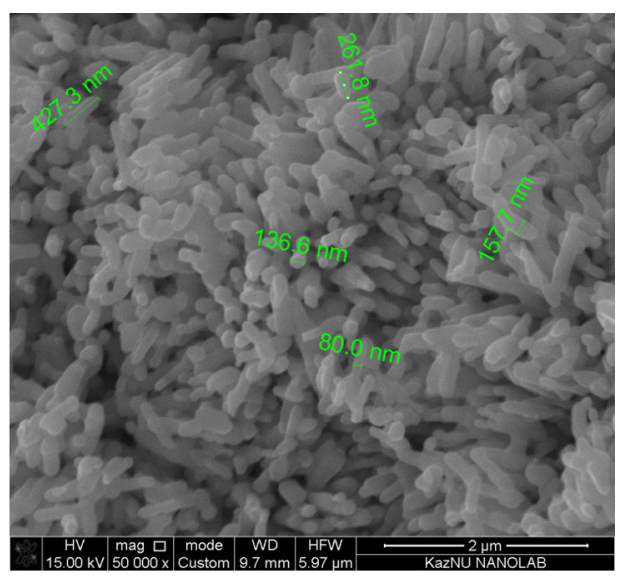

$b$

Fig. 2. Morphology of $\mathrm{ZnO}$ samples obtained during annealing of zinc acetate in the atmosphere, annealing for 2 hours at temperatures: $a-400{ }^{\circ} \mathrm{C}$ sample 1 , $b-700^{\circ} \mathrm{C}$ sample 4

Table 2

$\mathrm{ZnO}$ particle characteristics

\begin{tabular}{c|c|c|c|c|c}
\hline № & Thickness $D, \mathrm{~nm}$ & Length $L, \mathrm{~nm}$ & average $L$ & average $D$ & $n=L / D$ \\
\hline 1 & $43-81$ & $300-539$ & 420 & 63 & 7 \\
\hline 2 & $54-88$ & $400-690$ & 545 & 71 & 8 \\
\hline 3 & $48-88$ & $400-688$ & 544 & 66 & 8 \\
\hline 4 & $80-136$ & $260-400$ & 330 & 98 & 3 \\
\hline 5 & $108-113$ & $109-358$ & 234 & 111 & 2 \\
\hline
\end{tabular}




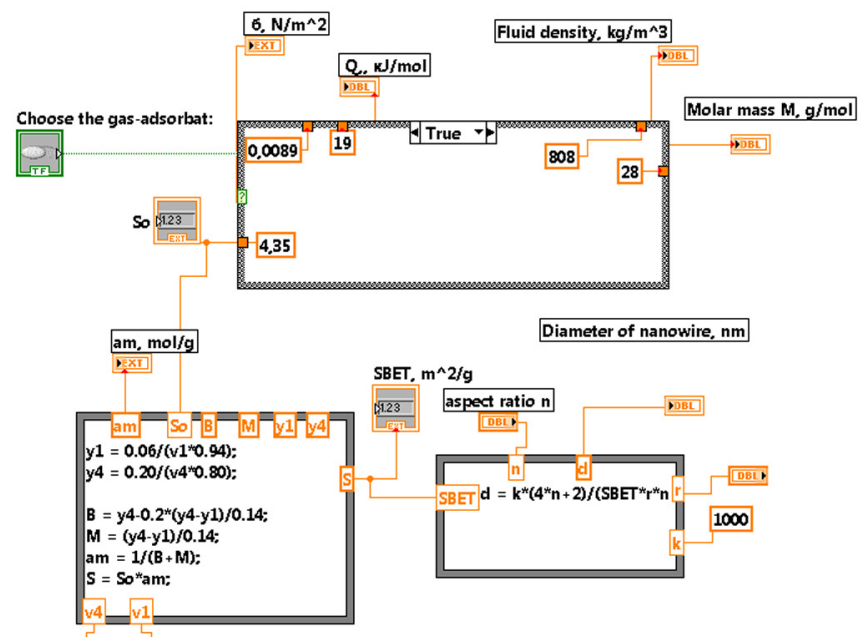

Fig. 3. A fragment of a program's block diagram for selecting an adsorbate gas, calculating the specific surface area and determining the size of nanorods according to sorption analysis

As can be seen from Table 3, the diameter of the rods will vary from 50 to $190 \mathrm{~nm}$, and its height - from 0.4 to 0.7 microns, depending on the conditions of receipt. It was noted that the height and diameter of the rods, determined by SEM and sorption data, differ significantly for some samples. One possible reason is the recrystallization of the material during annealing. It is likely that the annealing process changed the density of the material, which was the reason for the discrepancy between the data of sorption analysis and SEM for 2, 4, and 5 samples. Another reason may

\section{Conclusions}

Series of photocatalytically active zinc oxide samples were studied. According to the data of scanning electron microscopy, it was found that the particles have a rodshaped shape, while the heat treatment conditions significantly affect the size and aspect ratio of the nanorods. A program has been developed that automates the process of calculating the size of nanorods based on sorption analysis data. The program can also be used to analyze nanoparticles of a dif-

ferent shape, however, keep in mind, that several assumptions are the basis of the calculations: all particles are the same in size and shape (if it is, for example, the shape of nanorods, then when calculating their diameter it is assumed that the aspect ratio of all the rods in the sample are the same); particles have no internal pores and cavities. The program can be used for approximate express analysis of particle sizes and in the educational process. 


\section{References}

1. Ualikhanov RE, Kedruk EYu, Gritsenko LV, Chichero J, Abdullin HA. [Photocatalytic properties of nanostructured zinc oxide.] Vestnik KazNRTU. 2019;133(3):542. Russian

2. Labuayai S, Promarak V, Maensiri S. Synthesis and optical properties of nanocrystalline $\mathrm{ZnO}$ powders prepared by a direct thermal decomposition route. Applied Physics A. 2009;94:755.

doi:10.1007/s00339-008-4984-2

3. Belorus AO, Maraeva EV, Spivak YuM, Moshnikov VA. The study of porous silicon powders by capillary condensation. Journal of Physics: Conference Series. 2015;586:012017.

doi:10.1088/1742-6596/586/1/012017 